\title{
Caring for Patients with Rectal Cancer During the COVID-19 Pandemic
}

\author{
Kinga B. Skowron ${ }^{1} \cdot$ Roger D. Hurst ${ }^{1} \cdot$ Konstantin Umanskiy ${ }^{1} \cdot$ Neil H. Hyman ${ }^{1} \cdot$ Benjamin D. Shogan ${ }^{1}$
}

Received: 14 April 2020 / Accepted: 5 May 2020 / Published online: 15 May 2020

(C) 2020 The Society for Surgery of the Alimentary Tract

\begin{abstract}
The extraordinary spread of the novel coronavirus (COVID-19) has dramatically and rapidly changed the way in which we provide medical care for patients with all diagnoses. Conservation of resources, social distancing, and the risk of poor outcomes in COVID-19-positive cancer patients have forced practitioners and surgeons to completely rethink routine care. The treatment of patients with rectal cancer requires both a multidisciplinary approach and a significant amount of resources. It is therefore imperative to rethink how rectal cancer treatment can be aligned with the current COVID-19 pandemic paradigms. In this review, we discuss evidence-based recommendations to optimize oncological outcomes during the COVID-19 pandemic.
\end{abstract}

Keywords Rectal cancer · COVID-19 · Coronavirus

\section{Introduction}

Surgery remains the cornerstone of treatment for rectal cancer. Early in the COVID-19 pandemic, anecdotal reports and our own experience suggested disastrous complications in patients not recognized to have coronavirus who underwent seemingly routine operations. ${ }^{1}$ Yet while the possibility of occult asymptomatic COVID-19 is a threat, the inability to receive necessary treatments in the course of routine cancer care puts patients at increased risk for cancer-related morbidity and complications. $^{2}$ It is therefore appropriate that rectal cancer care algorithms be rethought and recommendations adjusted in the setting of this pandemic. During these challenging times, a broader lens must be employed with careful attention to patient safety, caregiver safety, and resource utilization. In this review, we explore the resources necessary for the care of patients with rectal cancer and discuss recommendations for

Benjamin D. Shogan

bshogan@surgery.bsd.uchicago.edu

1 Department of Surgery, The University of Chicago Medicine, 5841 S. Maryland Ave., Rm J557F, MC5095, Chicago, IL 60637, USA safely modifying rectal cancer treatment plans during this unprecedented time.

\section{The Risk of Morbidity and Mortality in Patients Undergoing Surgery During the COVID-19 Pandemic}

Data from China has demonstrated that patients with cancer are at a significantly higher risk of contracting the virus. ${ }^{3}$ Whereas the prevalence of cancer in the general population is $0.29 \%$, more than $1 \%$ of COVID-19positive patients had malignancy. Cancer patients were not only more likely to be infected but also suffered a more severe course, with an increased incidence of intensive care resource utilization, invasive ventilation, and mortality. While the data is still evolving, recent surgery (within 1 month of infection) appears to put cancer patients at even higher risk of postoperative morbidity and mortality, with $75 \%$ of patients suffering a severe course of COVID-19. Importantly, this increased morbidity may occur in asymptomatic patients, who were not known to be coronavirus carriers. Given that widespread coronavirus testing is limited and that the sensitivity in patients without symptoms remains poor, every rectal cancer patient must be assumed to be an asymptomatic carrier. ${ }^{1}$ 


\section{The Surgical Treatment of Rectal Cancer Requires Utilization of Critical Hospital Resources}

\section{In the Operating Room}

Rectal cancer surgery typically requires a major operation with substantial resource utilization. The mean operating time to perform a proctectomy is $262 \mathrm{~min}$ for laparoscopic and 209 min for open cases, longer than almost all emergency general surgery procedures. ${ }^{4}$ These operations require a significant amount of personal protective equipment (often with multiple gown, glove, and mask changes), and general anesthetic equipment such as ventilators.

The risk of exposure to the surgical team should also be considered, as these operations require large amounts of staff, including multiple surgeons, nurses, operating room technicians, and anesthesia staff. Because the virus may be transmitted via respiratory secretions, there is significant risk of provider exposure during aerosolizing procedures, such as intubation and extubation. ${ }^{5}$ The virus has also been isolated from gastrointestinal secretions; although the transmissibility of viral products in stool is not well understood, there is at least theoretical risk of aerosolization of such particles by electrocautery during abdominal surgery. ${ }^{6-8}$

\section{Postoperative Care at the Hospital}

Even when enhanced recovery protocols that limit in-hospital resource utilization are used, patients will usually require a minimum of a 2 to 3 -day admission. ${ }^{9}$ While most patients will have an uncomplicated hospital course, a subset of patients will have an extended stay; may require admission to the intensive care unit; and may require critical hospital resources such as blood transfusion, interventional radiology, or even reoperation.

During hospitalization in the time of COVID-19, patients will be isolated from usual support systems. To minimize exposures, many hospital systems are limiting patient rounds, and therefore, a patient may not be seen by their operating surgeon, but rather an alternative designated in-house provider. Additionally, many hospitals have implemented strict visitor restrictions, and thus, it is likely the patient will be alone without the visitation of their family or friends. The psychosocial effects of this isolation, especially for patients who are recovering physically and emotionally from their cancer operation, may have short- and long-term implications. ${ }^{10,11}$

\section{Postoperative Care After Discharge}

Patients who undergo rectal cancer surgery typically require close postoperative follow-up owing to the high complication rate; even when performed during normal circumstances, 10
$15 \%$ will be readmitted. ${ }^{12,13}$ In-person visits for either clinic follow-up or home health nursing are severely restricted, and early diagnosis of a preventable complication may not be possible. Further, social isolation and shelter in place restrictions place inherent limitations on the support that may be needed to manage complications, such as surgical site infections.

Additionally, many patients will require an ostomy during their index operation. While ostomy education often begins preoperatively, education once the ostomy is established is critical for patient success. ${ }^{14}$ During this pandemic, enterostomal therapists may not be as readily available and concomitant education of the patient's support person(s) may be very challenging. Upon discharge, home health support and other resources may be difficult to arrange in the setting of strict social distancing mandates. ${ }^{15,16}$

\section{Safely Delaying Surgery for Rectal Cancer}

As described above, the cumulative resources required to perform a proctectomy are significant. Given the current climate in which medical resources are limited, coupled with the potential increased morbidity of surgery in COVID-19 patients, delaying rectal cancer surgery is likely prudent. Here we will discuss the ways in which rectal cancer surgery can be safely delayed, thus freeing up valuable hospital resources, while optimizing outcomes in patients with rectal cancer.

Many patients have already embarked upon a treatment plan (e.g., neoadjuvant chemoradiation) with anticipation of surgery in the near future, and reconsideration of these plans is indicated in the present environment. Of course, alterations of treatment plans must be made in a shared decision-making fashion. Just as we would prior to the pandemic, surgical consultation and treatment recommendations must incorporate patient's wishes and comfort with a treatment's balance of risks and benefits: acceptance of an ostomy, ability to adhere to a close follow-up schedule, and comfort with a possibly higher risk of recurrence with some treatment options.

Furthermore, when considering a deviation from a patient's treatment plan or the implementation of a novel plan, it is imperative to discuss the options in a multidisciplinary setting and reach an agreement with all involved providers. Given that chemotherapy is also not without risk and requires patients to come to the hospital, oncologist may opt for shorter courses of chemotherapy or oral options, treatment decisions which may result in a need for earlier surgery. Thus, reaching a mutual understanding regarding the risks to the patient and the overall utilization of the healthcare system is crucial.

\section{Stage I}

Although local excision may be offered to highly selected early-stage lesions, total mesorectal excision (TME) is often 
recommended, as the risk of lymph node metastasis is approximately $10-13 \% .{ }^{17}$ During the COVID-19 pandemic, patients with a $\mathrm{T} 1$ rectal cancer may be more liberally considered for local excision, either endoscopically or transanally. These procedures are generally much less resource-intensive than TME as they are typically performed on an outpatient basis and often without the need for general anesthesia. Patients with high-risk features (depth of submucosal invasion, lymphovascular invasion, and location in the lower third of the rectum) may undergo completion TME at a time considered safe from a COVID-19 perspective, or alternatively offered adjuvant chemoradiotherapy if they are unwilling to pursue TME. ${ }^{18,19}$ If operating room resources are such that there is a long delay to TME, such patients should be recommended chemoradiotherapy. Patients with low-risk T1 cancer may not require any further therapy. Patients with $\mathrm{T} 2$ rectal cancer have a 20-30\% risk of lymph node metastasis and are thus typically recommended to undergo TME, given that local excision results in a $30-40 \%$ risk of recurrence..$^{20,21}$

\section{Stage II or III}

Patients with locally advanced, T3-T4 or N+ disease, are most often recommended a multimodality treatment approach, consisting of neoadjuvant chemoradiotherapy and surgical resection, followed by adjuvant chemotherapy. ${ }^{19}$ But similar oncologic outcomes can be achieved with numerous alternative treatment combinations. These can be streamlined during the pandemic to optimize resource utilization and minimization of patient and provider contact.

The decision to pursue long-course versus short-course radiotherapy has resource utilization implications, although its oncologic efficacy is likely equivalent. While proponents of long-course radiotherapy promote its greater effects in terms of rectal tumor regression, multiple studies have demonstrated equivalence between short-course ( 25 Gy in 5 fractions once daily for 5 days) and long-course (1.8-2-Gy fractions 5 days per week for 5 weeks) radiation in terms of local control and disease-free survival. ${ }^{22,23}$ While oncologically similar, shortcourse therapy results in dramatically fewer encounters for patients ( 25 visits for long course versus 5 visits for short course), lowering risk of coronavirus exposure for both patients and clinicians. Furthermore, more patients may be treated if each patient requires fewer sessions. Therefore, shortcourse neoadjuvant radiotherapy is appropriately recommended. $^{24}$

Surgical dogma has often suggested that proctectomy should be performed 4-8 weeks following long-course radiotherapy and 1 week following short-course radiotherapy. However, recent analysis of the multicenter randomized Stockholm III trial demonstrated a better rate of pathologic complete response in patients treated with short-course radiotherapy with a 4-8-week delay before TME, than among those treated with traditional short course (followed by immediate surgery) or long course (followed by surgery 4-8 weeks later). ${ }^{25}$ Therefore, delaying surgery up to 8 weeks following short-course radiotherapy appears safe and may be recommended during the current COVID-19 pandemic. If longcourse radiotherapy is chosen (or for patients currently in treatment), a delay of more than $8-10$ weeks after completion of radiotherapy may result in worse outcomes. In the multicenter randomized GRECCAR- 6 study, a delay beyond 11 weeks increased surgical morbidity and resulted in compromised surgical margins (complete TME $78.7 \%$ versus $90 \% ; p$ $=0.0156$ ) without increasing the pathologic complete response rate. ${ }^{26,27}$ Therefore, if long-course radiotherapy is in the treatment regimen, surgery is best not delayed past 8 weeks if possible.

Although postoperative adjuvant chemotherapy is often considered the standard of care in patients with locally advanced disease, the concept of total neoadjuvant therapy (TNT) has gained traction in the USA, even prior to the COVID-19 pandemic. This strategy refers to the preoperative administration of a full course of systemic chemotherapy, either before or after neoadjuvant radiation. ${ }^{28,29}$ Benefits of this approach include improved tumor response, earlier administration of systemic therapy to address metastatic disease, and improved delivery of therapy, as postoperative patients with complications may not be able to receive chemotherapy.

One concern, though, is that early exposure to chemotherapy may result in toxicity which may negatively impact surgery or surgical recovery. In the RAPIDO trial, patients were randomized to total neoadjuvant therapy consisting of shortcourse radiation followed by chemotherapy and surgery versus neoadjuvant long-course radiation followed by surgery with or without adjuvant chemotherapy. Authors noted that all patients in the TNT arm received radiation and $84 \%$ of patients were able to complete at least $75 \%$ of the prescribed chemotherapy, compared with $94 \%$ completing radiation and only $57 \%$ completing recommended chemotherapy in the adjuvant chemotherapy arm. Forty-eight percent of patients in the TNT arm suffered treatment-related toxicity, versus $25 \%$ in the long-course arm. However, there was no difference in the surgical procedures performed or in perioperative morbidity between the two arms. Thus, it appears that TNT is a safe option for the optimal delivery of recommended therapies for rectal cancer. ${ }^{30}$

Perhaps the most relevant trial is the Timing of Rectal Cancer Response to Chemoradiation Consortium trial, in which neoadjuvant radiotherapy was administered, followed by $0,2,4$, or 6 cycles of chemotherapy (mFOLFOX6). ${ }^{31}$ Authors found that a longer duration of chemotherapy achieved greater rates of pathologic complete response, but unlike the findings in the GRECCAR-6 study, increased time after radiation was not associated with surgical complications. One theory is that chemotherapy may suppress the 
inflammatory response which otherwise results within a few weeks after radiotherapy. While TNT appears safe and will delay surgery by over 4 months, this must be balanced by the fact that patients must travel to infusion centers biweekly for 3-4 months, potentially increasing risk of exposure to COVID-19.

Patient response to neoadjuvant therapy may be used to consider alternative treatment options and determine optimal timing for surgery. Patients who upon re-staging have not demonstrated a tumor response are unlikely to benefit from further delay and surgery is indicated. Meanwhile, patients with a considerable but incomplete response may benefit from a 10-12-week delay as they may ultimately demonstrate a complete or near-complete response. $^{32,33}$ Patients who have a complete clinical response may be offered resection, or can be entered into a "watch and wait" trial protocol (ClinicalTrials.gov Identifier: NCT02008656). ${ }^{34,35}$ Results thus far are promising, with a local recurrence rate of about $25 \%$ at 2 years, compared with rates of $10 \%$ or less after TME; patients with local recurrence are able to undergo curative salvage surgery. We recommend that patients with evidence of clinically complete response be considered for a "watch and wait" protocol and followed closely by physical exam, endoscopy, and imaging. When deciding how to proceed following a near-complete or complete response, it must be emphasized that "watch and wait" as a treatment modality is still unproven, is not considered standard of care, and should only be offered by providers who are familiar with the stringent surveillance regimen that is required to detect an early recurrence.

Therefore, for locally advanced rectal cancer, we recommend a total neoadjuvant therapy approach, with consideration for "watch and wait" trial entry for patients with complete clinical response after therapy. Patients who have completed a short-course radiation followed by 6 months of chemotherapy and a 6-8-week rest may consider surgery, or consider further cycles of chemotherapy if surgery at that time is not possible or unsafe in the setting of COVID-19.

\section{Stage IV}

Patients with metastatic disease, as before COVID, must be considered for the possibility of cure and appropriateness of resectability; standard National Comprehensive Cancer Network guidelines should be followed during the pandemic. ${ }^{19}$ If patients are approaching the completion of therapy during the pandemic, the risk of further delay should be balanced with risk of coronavirus-related complications and the previously discussed challenges related to recovery. A multidisciplinary discussion is recommended with regard to the benefit of further cycles of suppressive systemic therapy in order to further postpone surgical treatment, depending on pandemic-related resources in the patient's geographic area.

Of course, any patient presenting with urgent complications of their cancer, such as obstruction or perforation, should have this issue addressed as appropriate. Stenting or simple diversion should be strongly considered for obstruction.

\section{Precautions if Proceeding with Surgery}

Some patients may reach the end of nonsurgical therapies and require surgery before the end of the pandemic. If surgery is essential, either open or laparoscopic or robotic surgery may be considered based upon surgeon comfort and expertise. ${ }^{6}$ Providers should take all possible precautions to prevent inadvertently exposing caregivers. These include minimizing the use of energy devices; using closed-circuit smoke evacuation devices; wearing enhanced PPE; and, during laparoscopy, insufflating to lower pressures and desufflating the abdomen with a closed smoke evacuation system. Postoperative patient encounters should be minimized and telehealth, virtual visits, or telephone calls should be used as much as possible. Additionally, earlier and more frequent postoperative virtual visits should be performed which would likely capture early complications, preventing emergency room visits and readmissions. Hospitals with experience and capacity for mobile application follow-up should utilize these resources to maximize virtual patient contact. ${ }^{36,37}$

\section{Looking Ahead}

As our ability to test patients improves, it may be prudent to test all patients preoperatively and consider delaying surgery for any asymptomatic carriers of coronavirus to prevent inadvertently triggering occult disease postoperatively. Of course, our abilities to test for either active disease or immunity are quickly evolving, and available resources should be assessed regularly.

While it remains unclear how long we will have to practice in this resource-scarce environment, we must also prepare for the second wave of need which epidemiologists say will likely arise. As our colleagues in Italy have documented, reductions in staffing and resource availability may result in diminished colorectal screening; future diagnoses of rectal cancer may occur at advanced stages. ${ }^{38}$ Data has shown that such delays in therapy also result in increased healthcare cost and utilization. $^{39}$ Thus, even after the pandemic ends, the backlog of patients who need care will strain a healthcare system just recovering from COVID-19. 


\section{Conclusion}

In this challenging time, we must streamline our treatment of rectal cancer patients to limit the spread of coronavirus to both patients and caregivers, and provide sound oncologic care while preserving resources for the current battle against coronavirus. We recommend maximizing neoadjuvant therapy and limiting surgical treatment during the COVID-19 pandemic.

Acknowledgments Thank you to Dr. Christian Fernandez for providing perspective and advice during preparation of this manuscript.

\section{Compliance with Ethical Standards}

All authors contributed to this manuscript under the definition of authorship.

Conflict of Interest The authors declare that they have no conflict of interest.

\section{References}

1. Aminian A, Safari S, Razeghian-Jahromi A, Ghorbani M, Delaney CP. COVID-19 Outbreak and Surgical Practice: Unexpected Fatality in Perioperative Period. Annals of Surgery. In press 2020.

2. Wang H, Zhang L. Risk of COVID-19 for patients with cancer. The Lancet Oncology 2020;21:e181.

3. Liang W, Guan W, Chen R, Wang W, Li J, Xu K, Li C, Ai Q, Lu W, Liang H, Li S, He J. Cancer patients in SARS-CoV-2 infection: a nationwide analysis in China. The Lancet Oncology 2020;21:335337.

4. Braga M, Frasson M, Vignali A, Zuliani W, Capretti G, Di Carlo V. Laparoscopic Resection in Rectal Cancer Patients: Outcome and Cost-Benefit Analysis. Diseases of the Colon \& Rectum 2007;50: 464-471.

5. Wu D, Wu T, Liu Q, Yang Z. The SARS-CoV-2 outbreak: what we know. International Journal of Infectious Diseases. In press 2020.

6. Vigneswaran Y, Prachand VP, Posner MC, Matthews JB, Hussain M. What is the Appropriate Use of Laparoscopy over Open Procedures in the Current COVID-19 Climate? Journal of Gastrointestinal Surgery. In press 2020.

7. Xiao F, Tang M, Zheng X, Liu Y, Li X, Shan H. Evidence for Gastrointestinal Infection of SARS-CoV-2. Gastroenterology. In press 2020.

8. Wölfel R, Corman VM, Guggemos W, Seilmaier M, Zange S, Müller MA, Niemeyer D, Jones TC, Vollmar P, Rothe C, Hoelscher M, Bleicker T, Brünink S, Schneider J, Ehmann R, Zwirglmaier K, Drosten C, Wendtner C. Virological assessment of hospitalized patients with COVID-2019. Nature. In press 2020.

9. Thiele RH, Rea KM, Turrentine FE, Friel CM, Hassinger TE, McMurry TL, Goudreau BJ, Umapathi BA, Kron IL, Sawyer RG, Hedrick TL. Standardization of Care: Impact of an Enhanced Recovery Protocol on Length of Stay, Complications, and Direct Costs after Colorectal Surgery. Journal of the American College of Surgeons 2015;220:430-443.

10. Nichols TR. Social Connectivity in Those 24 Months or Less Postsurgery. Journal of Wound, Ostomy and Continence Nursing 2011;38:63-68.

11. McMullen CK, Bulkley JE, Altschuler A, Wendel CS, Grant M, Hornbrook MC, Sun V, Krouse RS. Greatest Challenges of Rectal
Cancer Survivors: Results of a Population-Based Survey. Diseases of the Colon \& Rectum 2016;59:1019-1027.

12. Schneider EB, Hyder O, Brooke BS, Efron J, Cameron JL, Edil BH, Schulick RD, Choti MA, Wolfgang CL, Pawlik TM. Patient Readmission and Mortality after Colorectal Surgery for Colon Cancer: Impact of Length of Stay Relative to Other Clinical Factors. Journal of the American College of Surgeons 2012;214: 390-398.

13. Bliss LA, Maguire LH, Chau Z, Yang CJ, Nagle DA, Chan AT, Tseng JF. Readmission After Resections of the Colon and Rectum: Predictors of a Costly and Common Outcome. Diseases of the Colon \& Rectum 2015;58:1164-1173.

14. Colwell JC, Kupsick PT, McNichol LL. Outcome Criteria for Discharging the Patient With a New Ostomy From Home Health Care: A WOCN Society Consensus Conference. Journal of Wound, Ostomy and Continence Nursing 2016;43:269-273.

15. Cengiz B, Bahar Z. Perceived Barriers and Home Care Needs When Adapting to a Fecal Ostomy: A Phenomenological Study. Journal of Wound, Ostomy and Continence Nursing 2017;44:6368.

16. Walker CA, Rau LA, Green MP. Welcoming Home the Patient with a New Ostomy. Home Healthcare Now 2015;33:385-389.

17. Nascimbeni R, Burgart LJ, Nivatvongs S, Larson DR. Risk of Lymph Node Metastasis in T1 Carcinoma of the Colon and Rectum. Diseases of the Colon \& Rectum 2002;45:200-206.

18. Kwakye G, Curran T, Uegami S, Finne CO 3rd, Lowry AC, Madoff RD, Jensen CC. Locally Excised T1 Rectal Cancers: Need for Specialized Surveillance Protocols. Diseases of the Colon \& Rectum 2019;62:1055-1062.

19. Rectal Cancer. National Comprehensive Cancer Network. NCCN Guidelines Version 2.2020.

20. Garcia-Aguilar J, Mellgren A, Sirivongs P, Buie D, Madoff RD, Rothenberger DA. Local Excision of Rectal Cancer Without Adjuvant Therapy: A Word of Caution. Annals of Surgery 2000;231:345-351.

21. Sitzler PJ, Seow-Choen F, Ho YH, Leong AP. Lymph node involvement and tumor depth in rectal cancers: an analysis of 805 patients. Diseases of the Colon \& Rectum 1997;40:1472-1476.

22. Bujko K, Nowacki MP, Nasierowska-Guttmejer A, Michalski W, Bebenek M, Kryj M. Long-term results of a randomized trial comparing preoperative short-course radiotherapy with preoperative conventionally fractionated chemoradiation for rectal cancer. British Journal of Surgery 2006;93:1215-1223.

23. Ngan SY, Burmeister B, Fisher RJ, Solomon M, Goldstein D, Joseph D, Ackland SP, Schache D, McClure B, McLachlan SA, McKendrick J, Leong T, Hartopeanu C, Zalcberg J, Mackay J. Randomized trial of short-course radiotherapy versus long-course chemoradiation comparing rates of local recurrence in patients with T3 rectal cancer: Trans-Tasman Radiation Oncology Group trial 01.04. Journal of Clinical Oncology 2012;30:3827-3833.

24. Marijnen CAM, Peters FP, Rödel C, Bujko K, Haustermans K, Fokas E, Glynne-Jones R, Valentini V, Spindler K-LG, Guren MG, Maignon P, Calvo FA, Pares O, Glimelius B, SebagMontefiore D. International expert consensus statement regarding radiotherapy treatment options for rectal cancer during the COVID 19 pandemic. Radiotherapy and Oncology. In press 2020.

25. Erlandsson J, Lörinc E, Ahlberg M, Pettersson D, Holm T, Glimelius B, Martling A. Tumour regression after radiotherapy for rectal cancer - Results from the randomised Stockholm III trial. Radiotherapy and Oncology 2019;135:178-186.

26. Lefevre JH, Mineur L, Kotti S, Rullier E, Rouanet P, de Chaisemartin C, Meunier B, Mehrdad J, Cotte E, Desrame J, Karoui M, Benoist S, Kirzin S, Berger A, Panis Y, Piessen G, Saudemont A, Prudhomme M, Peschaud F, Dubois A, Loriau J, Tuech JJ, Meurette G, Lupinacci R, Goasgen N, Parc Y, Simon T, Tiret E. Effect of Interval ( 7 or 11 weeks) Between Neoadjuvant 
Radiochemotherapy and Surgery on Complete Pathologic Response in Rectal Cancer: A Multicenter, Randomized, Controlled Trial (GRECCAR-6). Journal of Clinical Oncology 2016;34:3773-3780.

27. Huntington CR, Boselli D, Symanowski J, Hill JS, Crimaldi A, Salo JC. Optimal Timing of Surgical Resection After Radiation in Locally Advanced Rectal Adenocarcinoma: An Analysis of the National Cancer Database. Annals of Surgical Oncology 2016;23: 877-887.

28. Cercek A, Roxburgh CSD, Strombom P, Smith JJ, Temple LKF, Nash GM, Guillem JG, Paty PB, Yaeger R, Stadler ZK, Seier K, Gonen M, Segal NH, Reidy DL, Varghese A, Shia J, Vakiani E, Wu AJ, Crane CH, Gollub MJ, Garcia-Aguilar J, Saltz LB, Weiser MR. Adoption of Total Neoadjuvant Therapy for Locally Advanced Rectal Cancer. JAMA Oncology 2018;4:e180071.

29. Ludmir EB, Palta M, Willett CG, Czito BG. Total neoadjuvant therapy for rectal cancer: An emerging option. Cancer 2017;123: $1497-1506$.

30. van der Valk MJM, Marijnen CAM, van Etten B, Dijkstra EA, Hilling DE, Kranenbarg EM, Putter H, Roodvoets AGH, Bahadoer RR, Fokstuen T, Ten Tije AJ, Capdevila J, Hendriks MP, Edhemovic I, Cervantes AMR, de Groot DJA, Nilsson PJ, Glimelius B, van de Velde CJH, Hospers, GAP; Collaborative investigators. Compliance and tolerability of short-course radiotherapy followed by preoperative chemotherapy and surgery for highrisk rectal cancer - Results of the international randomized RAPIDO-trial. Radiotherapy and Oncology 2020;147:75-83.

31. Garcia-Aguilar J, Chow OS, Smith DD, Marcet JE, Cataldo PA, Varma MG, Kumar AS, Oommen S, Coutsoftides T, Hunt SR, Stamos MJ, Ternent CA, Herzig DO, Fichera A, Polite BN, Dietz DW, Patil S, Avila K; Timing of Rectal Cancer Response to Chemoradiation Consortium. Effect of adding mFOLFOX6 after neoadjuvant chemoradiation in locally advanced rectal cancer: a multicentre, phase 2 trial. The Lancet Oncology 2015;16:957-966.

32. Perez RO, Habr-Gama A, São Julião GP, Gama-Rodrigues J, Sousa AH Jr, Campos FG, Imperiale AR, Lynn PB, Proscurshim I, Nahas $\mathrm{SC}$, Ono CR, Buchpiguel CA. Optimal timing for assessment of tumor response to neoadjuvant chemoradiation in patients with rectal cancer: do all patients benefit from waiting longer than 6 weeks?
International Journal of Radiation Oncology Biology Physics 2012;84:1159-65.

33. Petrelli F, Sgroi G, Sarti E, Barni S. Increasing the Interval Between Neoadjuvant Chemoradiotherapy and Surgery in Rectal Cancer: A Meta-analysis of Published Studies. Annals of Surgery 2016;263: 458-64.

34. Smith JJ, Strombom P, Chow OS, Roxburgh CS, Lynn P, Eaton A, Widmar M, Ganesh K, Yaeger R, Cercek A, Weiser MR, Nash GM, Guillem JG, Temple LKF, Chalasani SB, Fuqua JL, Petkovska I, Wu AJ, Reyngold M, Vakiani E, Shia J, Segal NH, Smith JD, Crane C, Gollub MJ, Gonen M, Saltz LB, Garcia-Aguilar J, Paty PB. Assessment of a Watch-and-Wait Strategy for Rectal Cancer in Patients With a Complete Response After Neoadjuvant Therapy. JAMA Oncology 2019;5:e185896.

35. van der Valk MJM, Hilling DE, Bastiaannet E, Meershoek-Klein Kranenbarg E, Beets GL, Figueiredo NL, Habr-Gama A, Perez RO, Renehan AG, van de Velde CJH; IWWD Consortium. Long-term outcomes of clinical complete responders after neoadjuvant treatment for rectal cancer in the International Watch \& Wait Database (IWWD): an international multicentre registry study. The Lancet 2018;391:2537-2545.

36. Agri F, Hahnloser D, Demartines N, Hübner M. Gains and limitations of a connected tracking solution in the perioperative follow-up of colorectal surgery patients. Colorectal Disease. In press 2020.

37. Wang QQ, Zhao J, Huo XR, Wu L, Yang LF, Li JY, Wang J. Effects of a home care mobile app on the outcomes of discharged patients with a stoma: A randomised controlled trial. Journal of Clinical Nursing 2018;27:3592-3602.

38. Pellino G, Spinelli A. How COVID-19 Outbreak Is Impacting Colorectal Cancer Patients in Italy: A Long Shadow Beyond Infection. Diseases of the Colon \& Rectum. In press 2020.

39. Delisle M, Helewa RM, Ward MAR, Hochman DJ, Park J, McKay A. The Association Between Wait Times for Colorectal Cancer Treatment and Health Care Costs: A Population-Based Analysis. Diseases of the Colon \& Rectum 2020;63:160-171.

Publisher's Note Springer Nature remains neutral with regard to jurisdictional claims in published maps and institutional affiliations. 